\title{
Measurement of the Glomerulus Diameter and Bowman's Space Thickness of Renal Albino Rats
}

\author{
Taras Kotyk ${ }^{1}$, Nilanjan Dey ${ }^{2}$, Amira S. Ashour ${ }^{3}$, Dana Balas-Timar ${ }^{4}$, Sayan Chakraborty, \\ Ahmed S. Ashour ${ }^{6}$, João Manuel R. S. Tavares ${ }^{7}$ \\ ${ }^{1}$ Ivano-Frankivsk National Medical University, Ivano-Frankivsk, UKRAINE. \\ Email: taras1390@gmail.com \\ ${ }^{2}$ Department of Information Technology, Techno India College of Technology, Kolkata, INDIA. \\ Email: neelanjan.dey@gmail.com \\ ${ }^{3}$ Dept. of Electronics and Electrical Communications Engineering, Faculty of Engineering, Tanta \\ Univ., EGYPT. CIT College, Taif University, KSA. Email: amirasashour@yahoo.com \\ ${ }^{4}$ Aurel Vlaicu University of Arad, Arad, ROMANIA. \\ Email: dana@xhouse.ro \\ ${ }^{5}$ Dept. of CSE, Bengal College of Engg.and Tech., Durgapur, West Bengal, INDIA. \\ Email: sayan.cb@gmail.com \\ ${ }^{6}$ Department of Human Anatomy \& Embryology, Faculty of Medicine, Tanta Univ., EGYPT. \\ Email: ahmedashour31@yahoo.com \\ ${ }^{7}$ Instituto de Ciência e Inovação em Engenharia Mecânica e Engenharia Industrial, Departamento \\ de Engenharia Mecânica, Faculdade de Engenharia, Universidade do Porto, Rua Dr. Roberto \\ Frias s/n, 4200-465 Porto, PORTUGAL. \\ Email: tavares@fe.up.pt
}

*Corresponding author: Amira S. Ashour

Email: amirasashour@yahoo.com

Phone no.: +966591859749

\begin{abstract}
Glomerulus diameter and Bowman's space thickness in renal microscopic images indicate various diseases. Therefore, the detection of the renal corpuscle and related objects is a key step in histopathological evaluation of renal microscopic images. However, the task of automatic glomeruli detection is challenging due to their wide intensity variation, besides the inconsistency in terms of shape and size of the glomeruli in the renal corpuscle. Here, a novel solution is proposed which includes the Particles Analyzer technique based on median filter for morphological image processing to detect the renal corpuscle objects. Afterwards, the glomerulus diameter and Bowman's space thickness are measured. The solution was tested with a dataset of 21 rats' renal corpuscle images acquired using light microscope. The experimental results proved that the proposed solution can detect the renal corpuscle and its objects efficiently. As well as, the proposed solution has the ability to manage any input images assuring its robustness to the deformations of the glomeruli even with the glomerular hypertrophy cases. Also, the results reported significant difference between the control and affected (due to ingested additional daily dose (14.6 mg) of fructose) groups in terms of glomerulus diameter (97.40 \pm $19.02 \mu \mathrm{m}$ and $177.03 \pm 54.48 \mu \mathrm{m}$, respectively).
\end{abstract}

Keywords: Renal diseases, Glomerular hypertrophy, Medical imaging, Image analysis, particles Analysis algorithm. 


\section{Introduction}

Kidney is a significant organ which controls homeostasis, water volume and electrolytes in blood, blood pressure. The foremost function of the kidney is urine filtration. Histologically, the kidney is formed of peripherally situated cortex and a centrally located medulla. The cortex is very rich in nephrons that considered the main functional unit consisting of the renal corpuscle and renal tubules. On histological specimens, renal corpuscle looks like a small rounded structure that has a centrally located glomerulus surrounded peripherally with Bowman's capsule $[1,2]$. The glomerulus is a collection of capillaries lined united by a delicate mesangial matrix [3-5]; while, the Bowman's capsule consists of two epithelium layers: visceral (envelops glomerulus) and parietal.

Glomerular hypertrophy is indicated by the glomerular diameter. It is related to abundant human diseases and in tentative animal models such as rats. Such diseases are, namely: the Focal segmental glumerlosclerosis (FSGS), Extensive loss of renal mass, Diabetes, Oligomeganephronia, Reflux nephropathy, Obesity/sleep apnea, and Unilateral renal agenesis evoke glomerular hypertrophy, which increases the glomerulus diameter [6]. These diseases affect the glomerular morphometry that justifies the developing of accurate solutions for the assessment of the glomerular diameter.

In addition, obesity, advanced glycation and lacking of the tetraspan in CD151 can lead to Bowman's capsule expansion that is caused by either glomerular hyper filtration, which is indicated by Bowman's capsule dimensions, or tubular cast formation. For example, kidney undergoes numerous significant structural and metabolic changes with obesity, including glomerular hyper filtration, thickening of the glomerular basement membrane, and thickening of the mesangial matrix, proliferation of mesangial cells and the expansion of Bowman's capsule. In humans, micro albuminuria is the earliest clinical obesity manifestation related to the kidney damage and diabetic nephropathy, is characterized by glomerulosclrosis.

Furthermore, there are common factors that can affect the glomerular filtration and solute excretion on the kidney such as the aging and diet type. Thus, the pathological changes in the renal corpuscle of animal disease models supply significant information in screening composites to target such diseases.

Thus, various diseases can affect the renal corpuscle which is manifested by changing of corpuscles morphometric parameters such as glomerulus diameter and Bowman's capsule dimension. Accurate measuring of these parameters in experimental animal models assists the early diagnosis of such renal diseases and helps to prognosis kidney function changes. However, manual measurements consume time, increase the probability of miscalculations and are prone to errors. Furthermore, the common variations of the Bowman's capsule dimensions and glomerulus diameter, limit the measurements performed by the pathologist and lead to incorrect diagnosis.

Generally, object detection algorithms have two particular points of research in the case of renal corpuscle detection, namely: the non-rigid shapes of the objects in the microscopic images and high variety of the glomerulus intensity in the images under analysis. Typically, sliding window techniques have been used to detect the glomerulus at each location in the renal images [7-9]. While, standard parametric active contour algorithms are suitable for object tracking, they fail to track multiple objects particularly when the objects are close to each other.

Since, the detection of the objects' particle size in binary image is a very significant issue that can be performed using particles analysis algorithm for image analysis or image processing via the analysis of particles. This technique is employed for particle detection and measurements 
using digital imaging. Such measurements include particle shape (morphology or shape analysis), particle size, as well as particles distribution.

Therefore, the goal of this study is to achieve high accurate detection of the renal corpuscle and its objects in light microscopy images of control and affected rats' renal images. Therefore, it was focused on the automated detection and measurement of renal corpuscles objects employing a new solution based on the particles analyzer in binary images. Thus, the RGB original images are split into three channels from which the green channel is used as it contains the main information about the renal corpuscle. In addition, the proposed solution is able to detect glomerulus hypertrophy that leads to Bowman's capsule with interrupted space.

The remaining manuscript is structured as follows. Next section presents the related work. Section 3 includes the proposed solution and describes the two phases used for the detection and measurement of the glomerulus diameter and the Bowman's capsule dimension. The results are discussed in section 4 , followed by the conclusions in section 5 .

\section{Related work}

Analysis of renal microscopic sample images is an imperative tool for several disease diagnoses. Although quantitative analysis of the renal capsule objects is crucial in pathologist studies, only inadequate numbers of consistent techniques have been proposed. Existing solutions for glomerulus aided automatic analysis in microscopy renal images were discussed in [10]. Earlier, Osawa et al. [11] developed solutions using combined manual methods that can be applied to limited image datasets; however, they are very time consuming and prune to visual errors. Few image processing methods have been proposed to detect and analyze the glomerular basement membrane (GBM). Ong et al. [12] suggested a method that measured the smoothness, intensity difference, uniformity and intensity continuity to track the GBM centerline based on region growing. The evaluation of the results was performed by the pathologist.

In [13], a Rectangular Histogram of Oriented Gradient (R-HOG) was suggested as a potential candidate descriptor for glomerulus objects detection. It had the ability to capture the information of the gradient magnitude in the image under analysis. Therefore, it is robust to the changes in intensity caused by the heterogeneity of the stained levels. The results showed poor detection performance resulting in some false positives and low recall. Using R-HOG descriptors obtained from both the magnitude and the direction of the gradient, the glomeruli detection performance resulted in recall values high enough to be useful in pathological evaluation. In [14], a method based on morphological image processing and active contour models (ACM) for the semiautomatic segmentation and analysis of the GBM was proposed for transmission electron microscopy (TEM) images. The comparative analysis involving the manual measurements of an experienced renal pathologist showed an error in the range of $36 \pm 11 \mathrm{~nm}$. Kamenetsky et al. [15] introduced techniques for GBM thickness semiautomatic analysis in TEM renal biopsy images. The obtained results compared to manual measurements (by an experienced renal pathologist) designated that the average and standard deviation of the differences in the mean widths provided by the split-and-merge (SAM) algorithm were $12 \pm 9 \mathrm{~nm}$.

Recently, in [16] a novel descriptor known as a segmental histogram of oriented gradients (HOG) was introduced to perform comprehensively the glomerulus detection in images of entire kidney sections. It utilized flexible blocks that can be adaptively fitted to the input images, which achieved robustness to the glomeruli deformations. The experiments using real image data expose that Segmental HOG attained significant enhancements in terms of detection performance in comparison to Rectangular HOG. Various detection techniques that have been used in several applications, as the ones suggested in [17- 21], can be potentially incorporated in the glomeruli detection. 
Generally, particle size of objects in binary image is a vital aspect in binary images. Wei et al. [22] proposed a novel approach to detect the particle size of objects based on binary image analysis. A difference sum template was employed to extract the object's edge, then a half-8connected domain algorithm was used to determine the coordinates set that corresponds to each object. Finally, the particle size of every object was evaluated through rectangular segmentation. The experimental results proved the accuracy in determining the particle sizes of objects in binary image rapidly.

Typically, in images presenting bulky intensity variability, color/textures leads to the dependence on the object's shape to correctly detect significant objects in histological images. Active contours attached to single objects have been proposed to resolve shape constraints. However, extending this technique into multi-objects is considered complex and an unresolved goal. Consequently, the proposed solution has been developed to deal with circular objects more effectively in a fully automatic framework. In resume, it allocates better identification of round shape objects in renal histological images, using particles analysis algorithm.

\section{Material and methods}

In this work, a novel proposed system to determine the glomerulus diameter and Bowman's space thickness of albino rat's kidney in colored stained specimens' images acquired by light microscopy has been developed. The proposed solution was implemented using Image J v. 149v software [http://imagej.nih.gov] and uses commands and macro language functions.

Since, fructose has potentially harmful effects on metabolism. It plays a vital role in the aging process, the pathogenesis of the vascular, renal and obesity [23]. Thus, the current experimental study data set consists of: i) control group (healthy rats) that received normal diet (62\% starch, $23 \%$ protein, $4 \%$ fat, $7 \%$ cellulose, standard vitamins and salt mixture) and ii) affected group due to receiving additional daily dose $(14.6 \mathrm{mg})$ of fructose solution for 30 days that affects the renal function. In this current experimental study, selected rats' samples of aged 2 months (regardless their gender) are captured from a pool of 2-6 months aged rats.

As, kidney undergoes several significant structural and metabolic changes with obesity, including hyper filtration of the glomeruli, expansion of Bowman's capsule, and thickening of the glomerular basement membrane. Consequently, the glomerular size is one of the significant indexes to express the condition of the kidney.

Therefore, the main contribution of this study is to measure the glomerulus diameter and Bowman's space thickness of renal albino rats which are either control or affected. In the affected group, the additional fructose dose leads to renal dysfunction that affects the measured dimensions. This evaluates the novel proposed approach efficiency to measure the glomerulus diameter and bowman's space thickness in various cases.

Figure 1, illustrates the block diagram of the solution that consists of two main phases: i) Renal corpuscle and related objects detection, then ii) measurement of the glomerulus diameter and the Bowman's capsule thickness. 


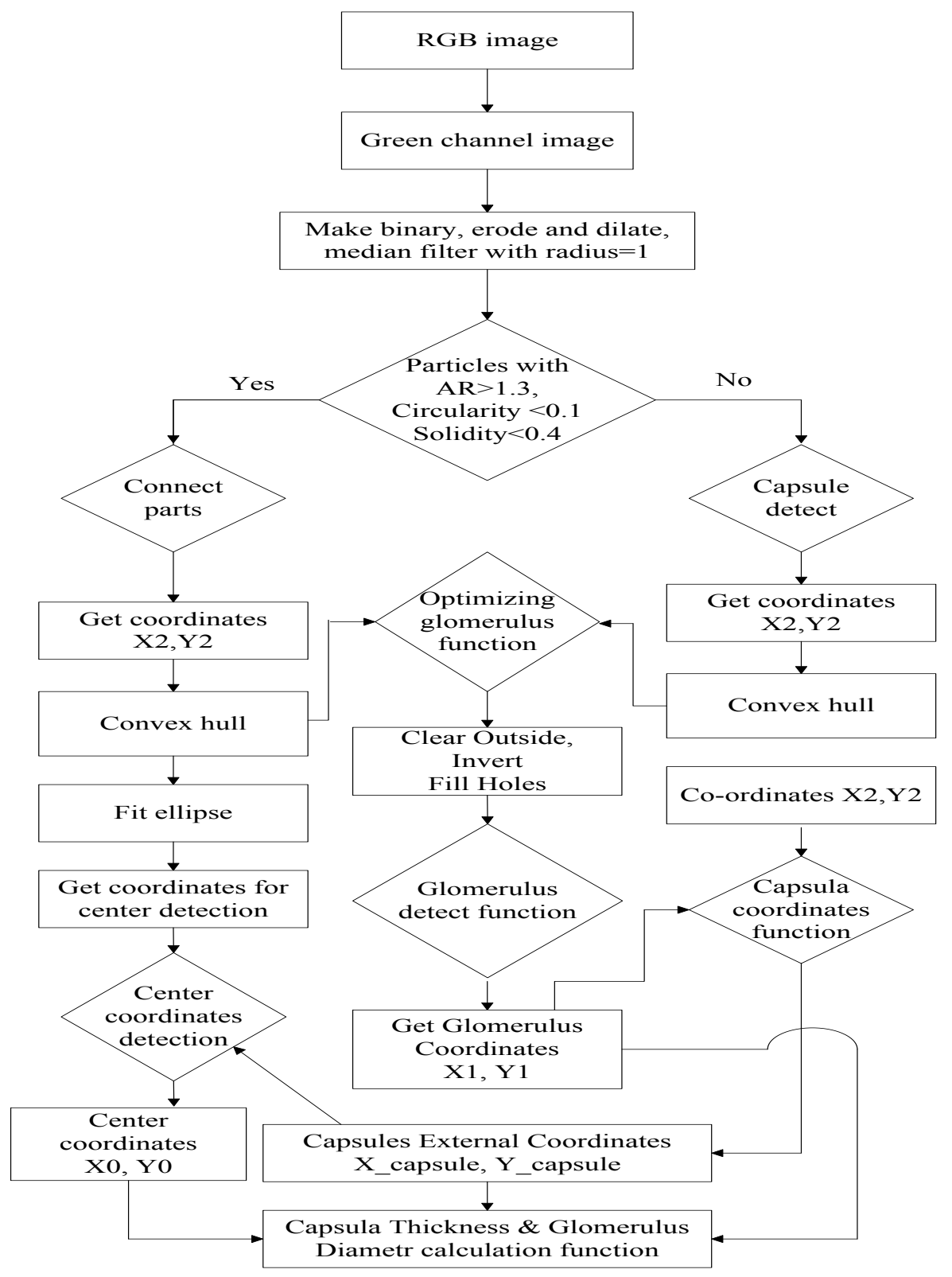

Fig.1Block diagram of the proposed system

Two phases for the proposed system are as follows.

\subsection{Phase I: Renal corpuscle detection}

Initially, the colored RGB (red-green-blue) input images are separated into red, green and blue channels. By investigating the resulting data in each of the three channels, it was found that the main relevant part of the renal corpuscle information appears in the green channel. While, the red channel contains information about the cell nucleus and a lot of noise is presented in the blue channel [24]. Thus, the green channel was selected to be processed by the solution developed. This requires image binarization as the ImageJ program for particles analysis is used to analyze objects on binarized images only. Thus, for detection and measurement, green channel images are converted into binary images before performing the analysis of the objects presented that leads to binary objects with rough boundaries. 
Consequently, filtering is required to attain smooth boundaries to specify the particles/ objects. The median filter is one of the simplest and superior filters that preserve edge structures than Gaussian smoothing. In addition, it has several advantages, namely: i) it does not shift boundaries, ii) has less sensitivity to extreme values (outliers) and iii) has no reduction in contrast through its steps. Therefore, to smooth the boundaries of the objects, morphological erode/ dilate reverse binary operators and a median filter with radius value equal to one are employed. Subsequently, the Particles Analyzer is used to analyze the objects, i.e. particles/structures, in the binary smoothed images and to identify the desired objects.

Through the Particles Analyzer shapes description, important values can be obtained. For example, the Aspect ratio (AR) is defined as the ratio between the major axis and the minor axis and has a minimum value equal to 1 . As the object under analysis becomes more elliptical, the $\mathrm{AR}$ value becomes greater. In the case of glomerular hypertrophy, the capsule splits into few parts that have very oblong shapes, but the smallest AR value should not be inferior to 1.3. This parameter is not enough robust to be used as unique descriptor. Generally, in all cases studied, the objects that are part of the renal corpuscle have circularity inferior to 0.1. Another descriptor is called solidity that indicates the ratio between the area of the object and the associated convex hull area. This parameter indicates that if the object is more like an arc, the convex hull area will be high, and the solidity value will be low. While, the circularity $=4 \pi^{*}$ area/perimeter ${ }^{\wedge} 2$. A value of 1.0 indicates a perfect circle. As the value approaches 0.0 , it indicates an increasingly elongated shape. Generally, there are three descriptors namely the AR, circularity, and solidity; when combined the proposed solution will be enough robust to describe the objects under analysis. When an object is detected, its boundary coordinates are stored and its Convex hull is built. While if there is no particle with solidity inferior to 0.4 based on trial-and-error method, the binarized image is inverted, and the Analysis particles algorithm starts to analyze the inverted image.

If the computational solution developed detects objects with Aspect ratio higher than 1.3, circularity lower than 0.1 and solidity lower than 0.4 , it indicates that these objects are part ofthe capsule and connect the partial boundaries of these objects, and the convex hulls of the found objects are built.

As shown in Figure1, the convex hull built for the capsule is then input to the optimizing glomerulus function, which performs removing and clearing all objects outside. This function is a command used in the particles analysis algorithm to assist ImageJ to identify the particle as glomerulus. It tries to optimize separation of particles from each other. Thus, implementation of the particles analysis ImageJ requires adjusting its settings to optimize the filtering down to a certain number of pixels. In addition, the image is inverted and the holes in the glomerulus object are filling. Essentially, ImageJ measures the area of the particles only (black pixels) that represents the region of interest (ROI) which shows the outer boundary. But, when measuring the ROI afterwards, the area of the hole will always be included in the result. Therefore, filling the holes measures the area without the holes. Consequently, when the image is binarized (Fig. 2C), glomerulus appears as white area with black particles inside it (they are the lumen of glomerulus vessels), While boundaries are the black particles of capsule. Afterwards, the image will be inverted after detection of particles that forms the capsule. The glomerulus will look like large black particle with white holes, which are the same lumens of the glomerulus vessels to be eliminated by "filling holes" to attain the glomerulus as in Fig. 2G.

The glomerulus detection function analyzes all objects presented in the image and detects the largest object to be determined as a glomerulus with boundaries coordinates stored in a vector $\left(\mathrm{X}_{1}, \mathrm{Y}_{1}\right)$.

Typically, $\left(\mathrm{X}_{2}, \mathrm{Y}_{2}\right)$ refers to the capsule boundary coordinates that include the external and internal parts. In addition, the internal parts indicate the glomerulus perimeter. Considering that $\left(\mathrm{X}_{1}, \mathrm{Y}_{1}\right)$ is the glomerulus boundary coordinates, the capsule coordinates function analyzes this 
array of coordinates and creates a new array for the capsule external perimeter coordinates. Generally, $\left(\mathrm{X}_{1}, \mathrm{Y}_{1}\right)$ is the internal coordinate of the capsule as well considered as the coordinates of the glomerulus perimeter. Meanwhile, (X_capsule,Y_capsule) refers to the capsule external perimeter coordinates. It is necessary for the corpusculum center coordinates detection and capsule thickness besides the glomerulus diameter calculation function, which is considered as the second phase of the proposed solution. Figure 2 depicts the steps of the $1^{\text {st }}$ phase implemented to detect the objects in the image under analysis that are indicated in Algorithm 1.

Algorithm 1:Renal corpuscle Detection

1. Read the original RGB image \% depicted in Fig. $2 \mathrm{~A}$

2. Get the Green channel image\% depicted in Fig. $2 B$

3. Convert the gray channel into binary image, use erode, dilate, and the median filter (with radius 1);\% depicted in Fig. $2 C$

4. Use the Analyzing function to detectthestructuresusing the conditions $A R>1.3$, Circularity $<0.1$, Solidity $<0.4$.

5. Get the coordinates of the capsule $X_{2}, Y_{2} ; \%$ depicted in Fig. $2 D$

6. Convex Hull \% depicted in Fig. $2 E$

7. Fit Ellipse. The corpuscle center coordinates $\left(X_{0}, Y_{0}\right)$ are detected according to the ellipse coordinates $\%$ depicted in Fig. $2 F$

8. Optimize the functions, clear outside, inverts and fills holes.

9. Run the glomerulus detection function to detect the glomerulus (green boundaries) and its coordinates $\left(X_{1}, Y_{1}\right) ; \%$ depicted in Fig. $2 G$

10. Detect the capsule coordinate function which is external boundary of capsule (red X_capsule, $Y$ _capsule) and which are boundaries of glomerulus (green); $\%$ depicted in Fig. $2 \mathrm{H}$

11. Determine the glomerulus diameter and capsule's thickness according to external boundary of capsule (X_capsule, Y_capsule), glomerulus boundaries $\left(X_{1}, Y_{1}\right)$ and corpusculum center $\left(X_{0}, Y_{0}\right)$ coordinates.\% depicted in Fig. $2 I$ 

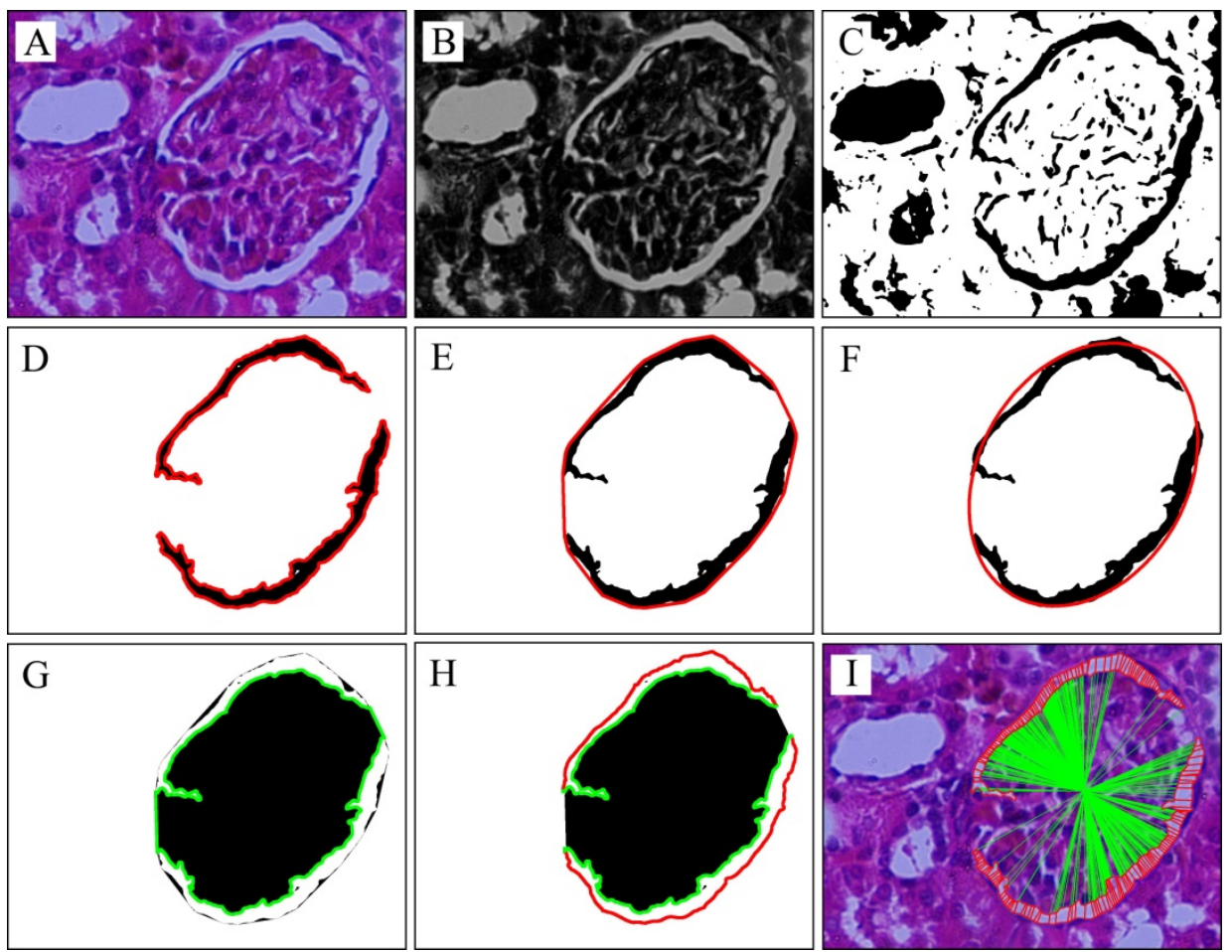

Fig. 2 Steps of the detection algorithm: (A) original RGB image, (B) green channel image, (C) binarized image, (D) detection of the capsule coordinates, (E) convex hull, (F) ellipse fitting, (G) detected glomerulus, $(\mathrm{H})$ detected external boundaries of the capsule (red) and boundaries of glomerulus (green), (I) glomerulus diameter and capsule`s thickness.

\subsection{Phase II: Glomerulus diameter and Bowman's space thickness measurement}

As mentioned above, the renal corpuscle is formed of a centrally located glomerulus that is surrounded peripherally by a Bowman's capsule. To measure the diameter of the glomerulus and thickness of Bowman's capsule it is required to determinate the coordinates of the corpuscle center.

The renal corpuscle center coordinates are calculated by considering the capsule external perimeter coordinates. The capsule has either a continuous perimeter or is divided into few parts. A "convex hull" operation connects the capsule that is divided into parts in the image. During the calculations, some "parts of the capsule" cannot be determined as being part of the capsule. Consequently, in this case, a "convex hull" selection is fitted into an ellipse. Therefore, the renal corpuscle center coordinates are estimated as the ellipse center that is used to calculate the diameter of the glomerulus.

Figure 3 illustrates the relation between the glomerulus (indicated by 1 ) and the perimeter of the renal corpuscle (indicated by 2) in Figure 3 (c and d). In addition, point $\mathrm{A}$ indicates the renal corpuscle center. These parameters are used to determine the capsule's thickness and its mathematical justification in Figure 3 ( $a$ and $b$ ).

Figures 3 (c and d), show the renal corpuscle's structure indicating that it is formed of a centrally located glomerulus radius and the Bowman's capsule thickness that is enclosed between the glomerulus perimeter and the capsule's external perimeter.

As illustrated in Figure 3 (a and b), the capsule's thickness $(\overline{\mathrm{BC}})$ is perpendicular to the glomerulus's perimeter tangent $(\overline{\mathrm{KL}})$. Also, the tangent $(\overline{\mathrm{KL}})$ is perpendicular to the glomerulus's radius $(\overline{\mathrm{AB}})$. As both $\overline{\mathrm{AB}}$ and $\overline{\mathrm{BC}}$ belongs to $\overline{\mathrm{AC}}$, thus the corpuscle radius $(\overline{\mathrm{AC}})$ is calculated as:

$$
\overline{\mathrm{AC}}=\overline{\mathrm{AB}}+\overline{\mathrm{BC}}
$$

Where, $\overline{\mathrm{AB}}$ is the glomerulus's radius and $\overline{\mathrm{BC}}$ is the capsule's thickness. Simultaneously, as illustrated in Figure 3b, $(\mathrm{ABC})$ does not represent a segment, but can be considered as a triangle, 
if the sum of the glomerulus's radius and the capsule's thickness is greater than the renal corpuscle radius:

$$
\overline{\mathrm{AC}}<\overline{\mathrm{AB}}+\overline{\mathrm{BC}}
$$

Based on this mathematical justification, the algorithm employed to measure the capsule's thickness and the glomerulus's radius is based on three parameters: 1) corpuscle's geometrical center coordinates; 2) capsule's external perimeter coordinates; 3) glomerulus's perimeter coordinates, as illustrated in Figure $3 b$.

$\mathbf{a}$
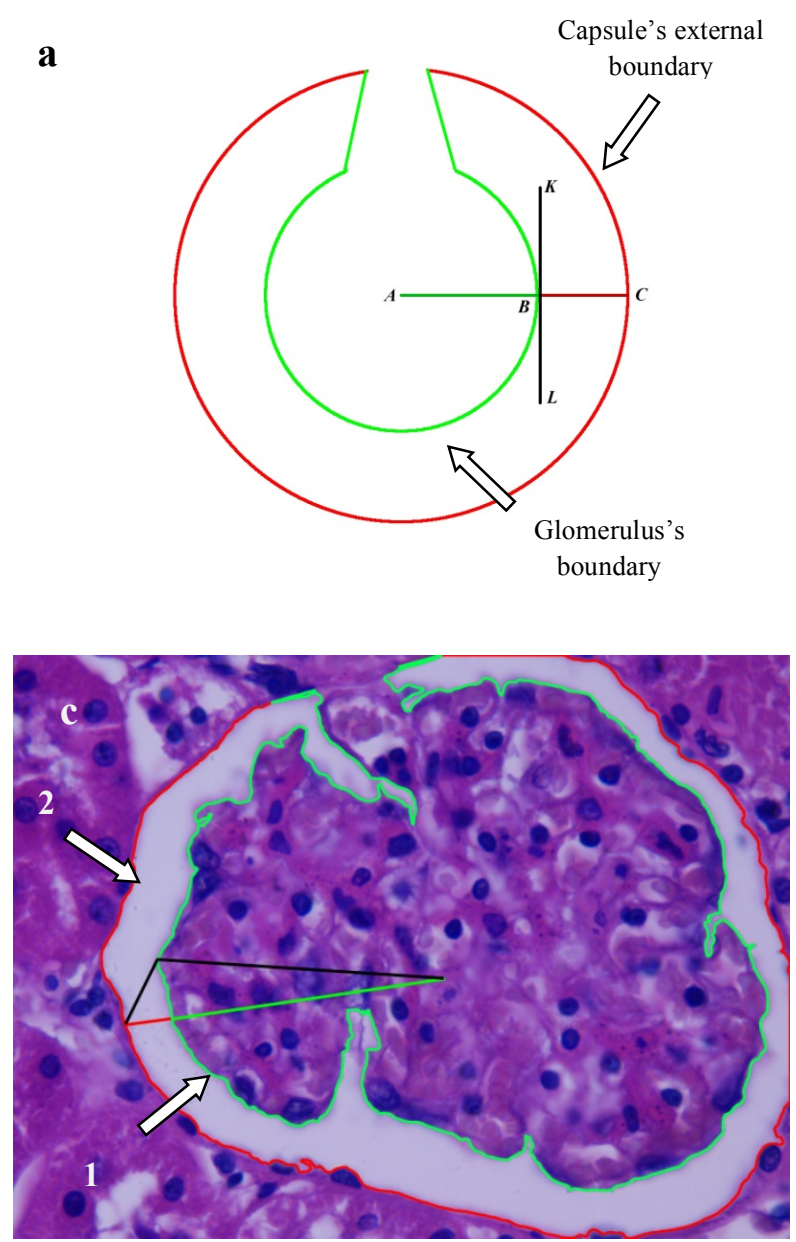

b
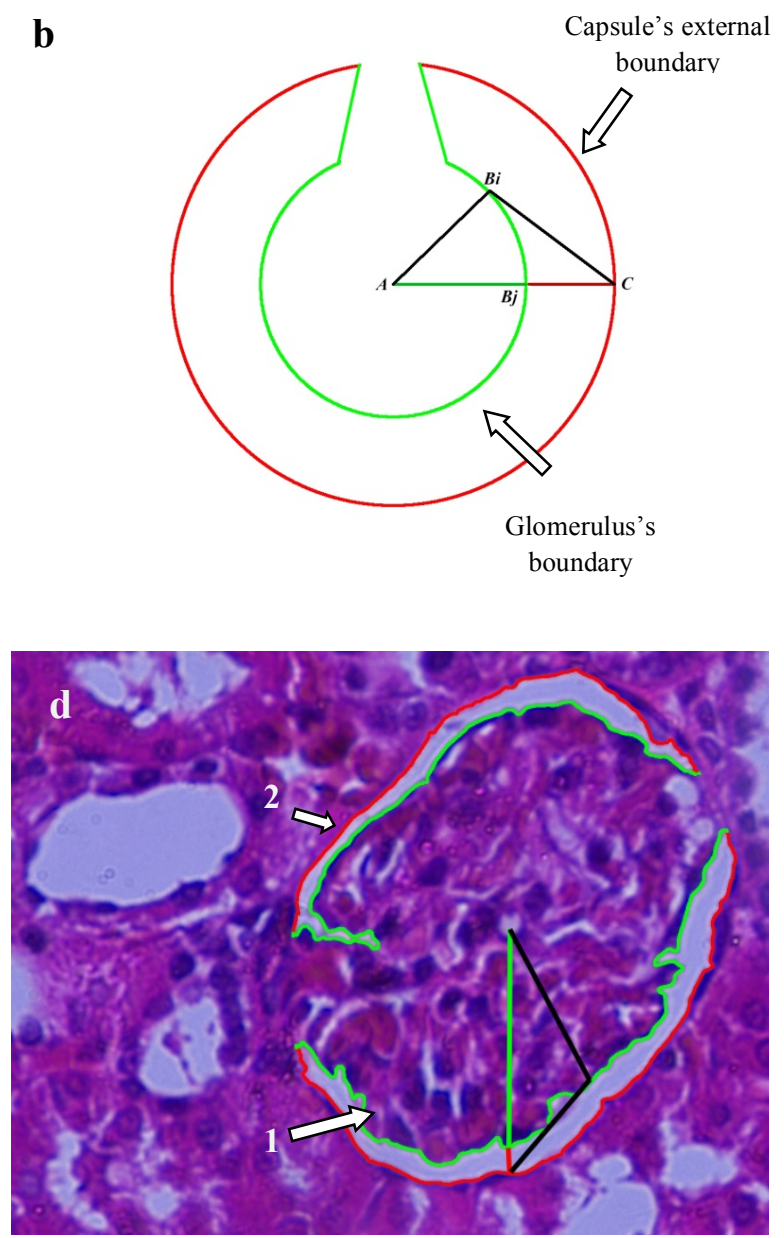

Fig. 3 The schema of the glomerulus's radius and capsule's thickness determination: $(a, b)$ is to the geometric representation of the glomerulus and the capsule, $(c, d)$ to the specimen with the interested boundaries marked, where: " 1 " indicates glomerulus's boundaries; "2" indicates the capsule's external boundaries.

The algorithm used in the second phase of the solution developed to measure the capsule's thickness and glomerulus's radius is presented in Algorithm 2. 
Algorithm 2: Measurement of the Bowman'scapsule thickness and glomerulus's radius

1. Consider $A$ is a point on the corpuscles geometric center,

$C$ is a point on the Bowman's capsules external perimeter,

$B_{i}$ and $B_{j}$ are points on the glomerulus's perimeter,

2. Define Tas the capsule's thickness array,

$R$ as the glomerulus's radius array.

3. Let $£$ be the total number of points on the Bowman's capsule external perimeter,

$\Xi$ is the total number of points on the glomerulus's perimeter.

4. Generate initial zero valued arrays for $T$ and $R$.

5. while $C<£$ and $B<\Xi$
6. for $C=1: £$
$\%$ points on the outer perimeter
7. for $B=1: \Xi$
$\%$ points on the inner perimeter

Determine the distance $\overline{A C}$ (from point " $A$ " to point " $C$ ”)

Determine the distance $\overline{A B}_{i}$ (from point " $A$ " to point " $B_{i}$ ")

Measure the distance $\overline{C B}_{i}$

Calculate the sum of $\overline{A B}_{I}$ and $\overline{C B}_{i}$

8. if $\overline{A B}_{i}+\overline{C B}_{i}>\overline{A C}$

$$
T=T ; R=R
$$

9. else if $\overline{A B}_{i}+\overline{C B}_{i}=\overline{A C}$

10.set $B_{j}=B_{i} \quad \%$ means points $A, B_{j}$ and $C$ belong to $\overline{A C}$

11. $T=T+C B_{j} \%$ means: $C B_{j}$ is added to the wall's thickness values)

12. $R=R+A B_{j} \%$ means: $A B_{j}$ is added to the glomerulus's radius values)

13. end else

14. end if

15. end for $B$

16. end for $C$

17. end while

Total Bowman'scapsule thickness = mean $(T)$;

Glomerulus's radius $=$ mean $(R)$;

Glomerulus's diameter $=2 *$ glomerulus's radius 


\section{Results and Discussion}

The experimental work was performed using a dataset of renal rat images obtained in the Anatomy Dept. Laboratory, Faculty of Medicine, Tanta University, Egypt. The tested specimen images were acquired using a light microscope with a magnification of X1000). In this current experimental study, selected albino rats' samples of aged 2 months (regardless their gender) are captured from a pool of 2-6 months aged rats. The dataset was a mix of both control and experimental affected groups ( 6 and 15 elements, respectively

In 1926, Arataki [25] reported the normal healthy Albino rats' glomerulus diameter at different ages. Several authors state that the glomeruli's diameter varies due to many factors, including:

- Their location, in the cortex: those located at the periphery have a smaller diameter than those located centrally,

- Gender: after fifty days from birth, the glomeruli's diameter in male is greater than that in female,

- Age: there is a comparatively rapid growth in the glomeruli's diameter from birth to 150 days, followed by a period of slower growth from 150 to 500 days.

In [25], the results regarding the glomerulus's diameter of albino rats are indicated. From the data reported, it can be established that the albino male rats of around 2 months age present a glomerulus's diameter between 77 and $85 \mu \mathrm{m}$, while the albino male rats of around 6 months age have between 151 and $121 \mu \mathrm{m}$ glomerulus's diameter. Additionally, the male rats of around 500 days age present glomerulus's diameters between 119 and $129 \mu \mathrm{m}$.

Consequently, to evaluate the performance of the proposed computational system, a comparison was performed between the obtained values by the system and the ones reported by Arataki. However, in this study the albino rats' samples of aged 2 months are selected from a pool of 2-6 months aged rats that corresponds to the period when the glomeruli's diameter change, since after this period the glomerulus's diameter is more or less constant [25].

In the experimental tests, the values used for the parameters of the particles algorithm related to the shape descriptors were: Aspect ratio $>1.3$, Circularity $<0.1$ and Solidity $<0.4$.

Figure 4 shows samples of one renal corpuscle per image at different conditions with the measured structures marked. Figures 4A and B show the control rat's specimen of 2 months age case. It is clear that the proposed system was able to accurately identify the renal corpuscle and its structures. The measures obtained for the glomerulus's diameter and the Bowman's capsule dimension are in line with the reported average normal values. The measured values for the glomerulus's diameter were $81.79 \mu \mathrm{m}$ and $87.63 \mu \mathrm{m}$, respectively. These values are very close to those reported in [25] for albino rats of 2 months age. While, the specimens in Figure 4C, is for an affected rat with a dilated Bowman's capsule value of $9.10 \mu \mathrm{m}$, which stills within the range for normal glomerulus's diameter values.

Figure 4D shows the Bowman's capsule that appears as one continuous part (unit), while Figure $4 \mathrm{E}$ indicates adhesions of the glomerulus with the Bowman's capsule due to glomerular hypertrophy with a diameter of $162.56 \mu \mathrm{m}$. Thus, in this case, the Bowman's space appears divided into parts and has a glomerulus's thicknesses of $5.80 \mu \mathrm{m}$. This proves the robustness of the proposed system as it has the ability to detect reformatted glomeruli in the glomerular hypertrophy cases.

Figure 4 (D - I) show cases of glomerular hypertrophy; from these, Figure 4 (F, H and I) are for dilated capsules with thickness of $9.21 \mu \mathrm{m}, 12.34 \mu \mathrm{m}$, and $9.84 \mu \mathrm{m}$, respectively. The same was established for the specimens shown in Figure 4 (F- I), associated to dilated Bowman's capsules as the thicknesses found are anomaly high. 

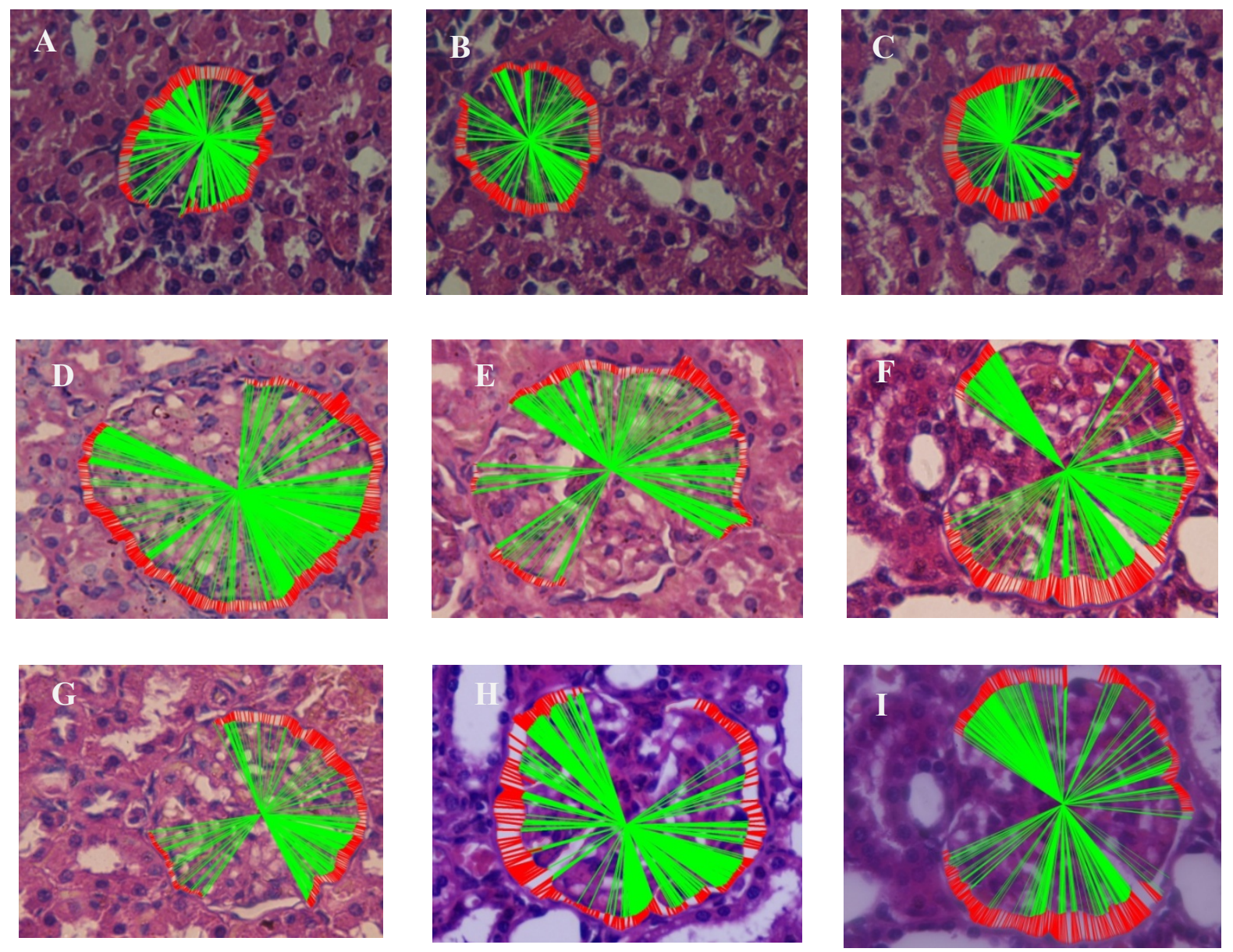

Fig.4 Histological light microscopic images for different cases of renal rat's capsule: A and B are of control healthy rats, $\mathrm{C}$ to I are of affected treated rats.

Figure 4 (A, B) demonstrates specimens of control rats aged two months illustrating rounded renal corpuscles with a centrally located glomerulus surrounded peripherally with Bowman's capsule. While, figure 4 (C-I) illustrates renal corpuscles with mild expanded Bowman's capsule, for the same two months aged rats that ingested fructose.

The proposed computational system was tested on 6 specimen images from the control group and 15 specimen images from the affected group. The obtained results are indicated in Figure 5.

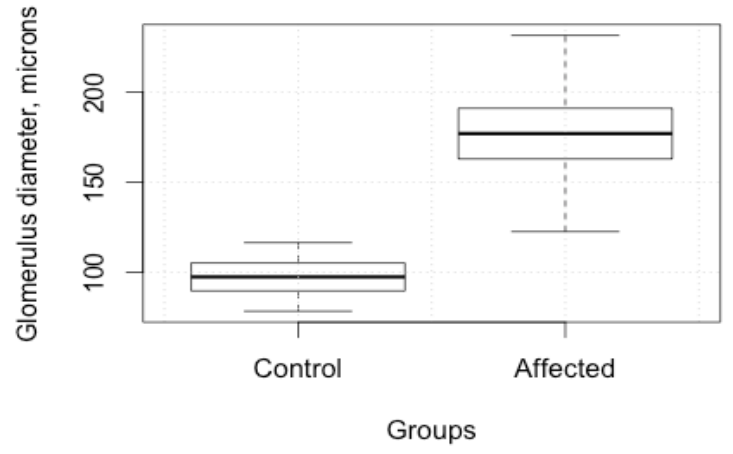

(A)

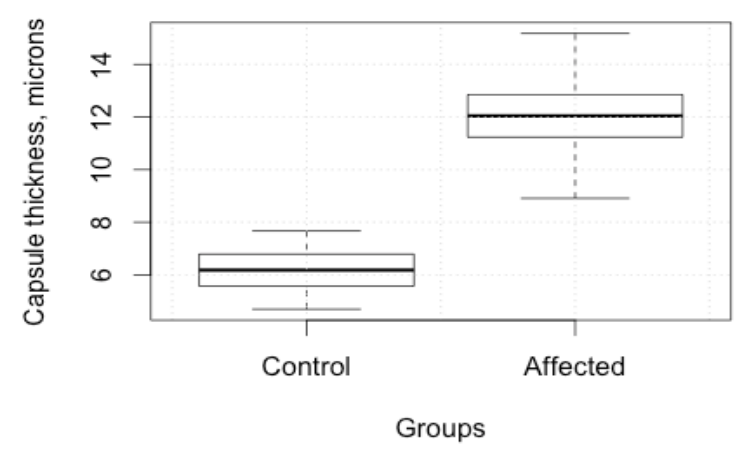

(B)

Fig. 5 Obtained values for the glomerulus diameter (A) and capsule's thickness (B) by the proposed system. 
Figure 5A shows that the glomerulus's diameter in the affected group has higher values than to the control group: $177.03 \pm 54.48 \mu \mathrm{m}$ for the affected group, and $97.40 \pm 19.02 \mu \mathrm{m}$ for the control group. A similar situation is found with the capsule's thickness, Figure 5B: $6.18 \pm 1.49$ $\mu \mathrm{m}$ for the control group, and $12.04 \pm 3.13 \mu \mathrm{m}$ for the affected group. In addition, Figure 5 reveals interesting information about the increment of the standard deviation in the case of the affected group.

Since, the significant difference value ( $p$-value) is a statistical significance used in health research to examine the difference between two groups in order to determine if it is statistically significant. Typically, it is determined by calculating the probability of error ( $p$-value). The difference between two groups (such as an affected versus control group) is evaluated to be statistically significant when $p=0.05$ or less. Such that at $p=0.05$, the differences between the two groups have only a $5 \%$ probability of occurring. While, at $\mathrm{p}=0.01$, the differences between the two groups have only a $1 \%$ probability of occurring. Thus, the values in Table 1 reveal that there was a significant difference of $(p<0.05)$ between the control and affected groups in terms of the measurement of the glomerulus's diameter and Bowman's capsule thickness.

Table 1 Comparative Measurement results

\begin{tabular}{|lcc|}
\hline & $\begin{array}{c}\text { Glomerulus's diameter } \\
\text { measurement } \\
\text { Control group }(\mathbf{n}=\mathbf{6})\end{array}$ & $\begin{array}{c}\text { Bowman's space } \\
\text { thickness }\end{array}$ \\
Affected Group $(\mathbf{n}=\mathbf{1 5})$ & $97.40 \pm 19.02$ & $6.18 \pm 1.49$ \\
Significant value $p$ & $177.03 \pm 54.48$ & $12.04 \pm 3.13$ \\
(Mann-Whitney-Wilcoxon Test) & 0.001622 & 0.000258 \\
\hline
\end{tabular}

Generally, the experimental results proved the efficiency of the proposed system to:

- Correctly detect the renal capsule and its structures, i.e. the glomerulus and Bowman's capsule;

- Measurement of the glomerulus's diameter and Bowman's capsule dimension;

- Correctly distinguish between control and affected rats' specimens cases;

- Detect and measure the renal corpuscle when it has multi-parts in the input image;

- Distinguish between control and affected groups with high significance, either when measuring the glomerulus's diameter or the Bowman's capsule thickness, which is required for proper diagnoses;

- Obtain measurement values that are in line with the ones reported by Arataki [25] and are commonly accepted in this domain.

- Robust and has the ability to detect reformatted glomeruli in the glomerular hypertrophy cases.

In the current study, the computed time complexity of the proposed system was studied using 21 histological images. It was found that the required computation time depends on the number of the selected points by the automated system in the boundaries of the glomerulus and Bowman's capsule as shown in Figure 6. 


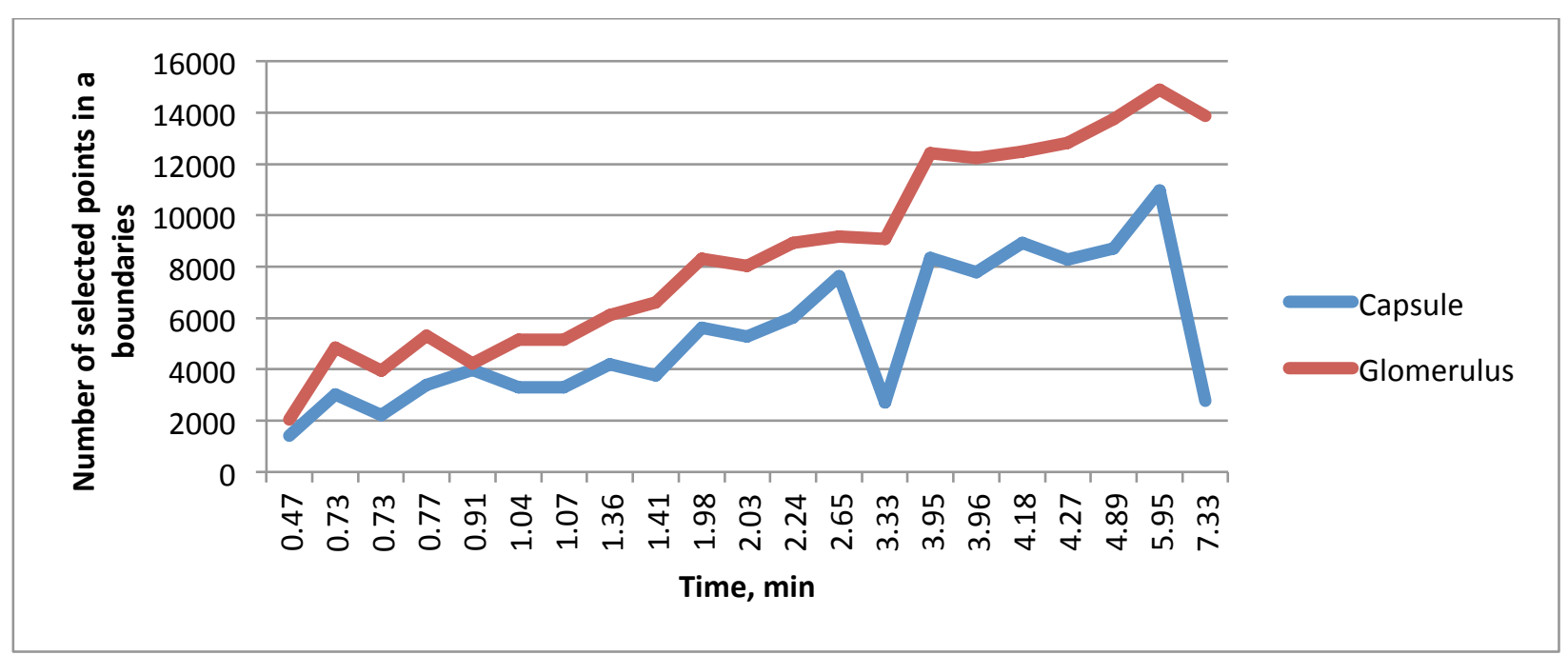

Fig.6 The time computation dependency on the number of selected points located on the boundaries of the glomerulus and Bowman's capsule.

Figure 6 depicts that the computational time is directly proportional to the number of points on the boundaries of the glomerulus and Bowmans capsule. By calculating the average time taken for all selected points on the boundary of all images, it is found that the detection of the coordinates of the external boundaries of the Bowman's capsule takes average time of $30 \%$ from the total time. While, the measurement phase of the glomerulus's diameter and Bowman's capsule thickness takes about $46 \%$ average time from the total time.

Glomerulus size is one of the imperative parameters to indicate the renal condition. Therefore, the main contribution of this study is to solve such problem by proposing a novel algorithm to identify/ detect the glomerulus followed by measuring the glomerulus's diameter and Bowman's capsule thickness. Lastly, the data set was a mix of both control and experimental affected groups that have renal failure due to the food type. Compared to the results obtained by Arataki, the proposed method proved its performance. The results established that both the glomerulus's diameter and the Bowman's capsule thickness increased in the affected group compared to the control one. In the present study, the renal rats' samples showed expanded in size Bowman's capsule due to ingested fructose.

\section{Conclusion and Future work}

Manual systems for determining the dimensions of renal corpuscle objects are very tedious, time consuming and biased mechanisms. Consequently, automated systems for the detection and accurate measurement of the glomerulus' diameter and Bowman's capsule thickness of renal albino rats are demanded.

The proposed system was tested on 6 and 15 images of control and affected albino rat groups, respectively. The experimental tests performed indicate $97.40 \pm 19.02 \mu \mathrm{m}$ for the glomerulus's diameter in the control group. The glomerulus's diameter increased in the affected group:177.03 $\pm 54.48 \mu \mathrm{m}$. On the other hand, the Bowman's capsule thickness increased from $6.18 \pm 1.49 \mu \mathrm{m}$ in the control group to $12.04 \pm 3.13 \mu \mathrm{m}$ in the affected group. In both cases, significant differences were achieved $(\mathrm{p}<0.05)$.

Comparatively to the values reported by Arataki [25] for the glomerulus's diameter, the values obtained by the proposed system in the control group are in agreement.

The solidity value is selected as 0.4 based on trial-and-error method. This selection is image/case specific. Therefore, as a future study this selection can be automated and studied.

The performance of the system proposed can be further improved by integrating more effective segmentation techniques. In addition, as illustrated by van Damme and Koudstaal in [26], and 
Arataki [25], the glomerular sizes are different between surface and juxta-medullary areas of the cortex. Accordingly, the measurement can be done in both areas and compared to each other.

\section{References}

[1] P.D. Vize, A.S. Woolf, J. Bard, The kidney, from normal development to congenital isease. London: Academic, 2003.

[2] M. Schwartz, J. Jennette, J. Olson, F. Silva, Pathology of the kidney. New York: Lippincott Williams \& Wilkins, 2007.

[3] A.C. Guyton, J.E. Hall, Textbook of Medical Physiology. Philadelphia: Elsevier Saunders, 2006, pp. 310.

[4] F.H. David, An Introduction to Human Physiology. Mc-Graw-Hill Medical, 2009, pp. 356370.

[5] A. Brandis, G. Blanch, E. Reale, U. Helmchen, K. Kuhn, Age dependent glomeruloselerosis and proteinuria occurring in rats of the Milan normotensive strain and not in rats of the Milan hypertensive strain. Lab Invest. 55(2) (1986) 234-43.

[6] A. Fogo, Glomerular hypertension, abnormal glomerular growth, and progression of renal diseases, Kidney International 57 (2000) S15-S21.

[7] C. Papageorgiou, T. Poggio, A trainable system for object detection. Int J Comput Vision 38(1) (2000) 15-33.

[8] S. Munder, D. M. Gavrila, An experimental study on pedestrian classification, IEEE Trans Pattern Anal Mach Intell 28(11)(2006)1863-1868.

[9] S. Maji, A. Berg, J. Malik, Classification using intersection kernel support vector machines is efficient. In IEEE CVPR, Anchorage, AK, 2008, pp. 1-8.

[10] J. Ma, J. Zhang, J. Hu, Glomerulus extraction by using genetic algorithm for edge patching. In IEEE CEC, Trondheim, Norway, 2009, pp. 2474-2479.

[11] G. Osawa, P. Kimmelstiel, V. Sailing, Thickness of glomerular basement membranes. Clinical Pathology 45 (1966) 7-20.

[12] S. H. Ong, S. T. Giam, Jayasooriah, R. Sinniah, Adaptive window-based tracking for the detection of membrane structures in kidney electron micrographs. Machine Vision and Applications 6 (1993) 215-223.

[13] N. Dalal, B. Triggs, Histograms of oriented gradients for human detection. In IEEE CVPR, IEEE Computer Society, Washington, DC, USA 1 (2005) 886-893.

[14] R. M. Rangayyan, I. Kamenetsky, H. Benediktsson, Segmentation and analysis of the glomerular basement membrane in renal biopsy images using active contours: a pilot study. Journal of Digital Imaging 23(3) (2010) 323-31.

[15] I. Kamenetsky, R. Rangayyan, H. Benediktsson, Analysis of the Glomerular Basement Membrane in Images of Renal Biopsies Using the Split-and-Merge Method: A Pilot Study, Journal of Digital Imaging 23(4) (2010) 463-474.

[16] T. Kato, R. Relator, H. Ngouv, Y. Hirohashi, T. Kakimoto, K. Okada, New Descriptor for Glomerulus Detection in Kidney Microscopy Image, Computer Vision and Pattern Recognition (2015) pp. 1-16.

[17] N. Dey, A. Das, Shape \& Size Analysis of Mineral Grains From photomicrographs Using Harris Corner Detection.International Journal of Advances in Engineering, Science and Technology (IJAEST) 2(2) (2012b).

[18] N. Dey, B. Nandi, A. B. Roy, D. Biswas, A. Das, S. S. Chaudhuri, Analysis of Blood Cell Smears using Stationary Wavelet Transform \& Harris Corner Detection. Published by Recent Advances in Computer Vision and Image Processing: Methodologies and Applications (2013)357-370. 
[19] N. Dey, M. Pal, A. Das, A Session Based Watermarking technique Within the NROI of Retinal Fundus Images for Authentication Using DWT, Spread Spectrum and Harris Corner Detection. International Journal of Modern Engineering Research 2 (3) (2012a) 749-757.

[20] N. Dey, A. B. Roy, A. Das, Detection and Measurement of Bimalleolar Fractures Using Harris Corner. ICACCI-2012 Chennai, India, 3-5 Aug. 2012c.

[21] T. Araki, N. Ikeda, F. Molinari, N. Dey, S. Bose, S. Shafique, A. El-Baz, et al.Automated and Accurate Carotid Bulb Detection, its Verification and Validation in Low Quality Frozen Frames and Motion Video. Int Angiol. 33(6) (2014) 573-89.

[22] L. Wei, C. Yuanyuan, W. Boxiong, Y. Chunyu, L. Jiannan, Fast Method to Detect Particle Sizes of Objects in Binary Image. 2013 Seventh International Conference on Image and Graphics, (2013) 252-268.

[23] A. Sheludiakova, K. Rooney, R.A. Boakes, Metabolic and behavioural effects of sucrose and fructose/glucose drinks in the rat, Eur. J. Nutr. (2011) 862-870 .

[24] J. Prinyakupt, C. Pluempitiwiriyawej, Segmentation of white blood cells and comparison of cell morphology by linear and naïve Bayes classifiers, BioMed Engineering OnLine 14(63) (2015) 1-19.

[25] M. Arataki, On The Postnatal Growth of The Kidney, With Special Reference to the Number and Size of The Glomeruli (Albino Rat).American Journal Of Anatomy 36(3) (1926).

[26] B. van Damme, J. Koudstaal, Measuring Glomerular Diameters in Tissue Sections. Virchows Arch. A Path. Anat. and Histol. 369( 1976) 283-291. 